\title{
Usefulness of the subunit principle in nasal reconstruction*
}

\author{
Felipe Bochnia Cerci ${ }^{1,2}$
}

DOI: http:/ /dx.doi.org/10.1590/abd1806-4841.20175278

\begin{abstract}
The subunit principle in nasal reconstruction proposed the concept of reconstructing the specific topographic subunits that were identified as dorsum, tip, columella, the paired alae, sidewalls, and soft triangles. In patients with more than $50 \%$ of subunit loss, removing the remaining portion of the subunit and reconstructing the entire subunit may yield better results. The placement of incisions along the borders of the subunits minimizes scar lines. Furthermore, as trapdoor contraction occurs, the entire restored subunit bulges in a way that mimics the normal contour of a nasal tip, dorsum or ala. Two cases of nasal reconstruction that followed this important principle are described.
\end{abstract}

Keywords: Mohs surgery; Nose neoplasms; Surgical flaps

\section{INTRODUCTION}

Since its description in 1985 by Burget and Menick, the subunit principle in nasal reconstruction has been incorporated as an essential step in preoperative planning. ${ }^{1}$ The principle popularized the technique of reconstructing the specific topographic subunits that were identified as dorsum, tip, columella, the paired alae, sidewalls, and soft triangles (Figure 1). In patients with more than $50 \%$ of subunit loss, Burget and Menick proposed removing the remaining portion of the subunit and reconstructing the entire subunit. This allowed placement of incisions along borders of esthetic subunits, minimizing scar lines.

Two cases that followed this important principle are described.

\section{CASE REPORT}

\section{Case 1}

A 42-year-old man presented to the Department of Dermatology with a nodular basal cell carcinoma on the right ala, excised by Mohs micrographic surgery. The resulting defect measured $1.6 \mathrm{x}$ $1.7 \mathrm{~cm}$ (Figure 2).
Since most of the subunit had been removed, the remaining subunit was excised and the defect was repaired with a nasolabial interpolation flap. Prior to flap execution, a cartilage graft was harvested from the ipsilateral antihelix/scaphoid fossa for better alar support. A defect template was created based on the contralateral ala, and demarcated on the nasolabial fold, connected to the pedicle (Figure 2). The flap was then incised, elevated and sutured into the primary defect. The nasolabial fold was closed primarily in three layers (Figure 2).

After three weeks, the patient was submitted to the second stage, which consisted on pedicle division and thinning of the flap. Nine months postoperatively, the patient had a good result with maintenance of function, alar lobule restoration, and alar crease preservation (Figure 3).

\section{Case 2}

A 72-year-old man presented to the Department of Dermatology with a mixed (infiltrative and nodular) basal cell carcinoma on the right ala. After two stages of Mohs micrographic

Work submitted on 22.10.2015

Approved by the Advisory Board and accepted for publication on 01.05.2016

* Work performed at the Department of Dermatology - Hospital Santa Casa de Curitiba

Financial support: None

Conflict of interest: None

Department of Dermatology (Mohs surgery) of Hospital Santa Casa de Curitiba - Curitiba (PR), Brazil.

Department of Dermatology (Mohs surgery) of Hospital de Clínicas da Universidade Federal do Paraná - Curitiba (PR), Brazil.

(C)2017 by Anais Brasileiros de Dermatologia 
surgery, clear margins were achieved resulting in a $2.7 \times 2.5 \mathrm{~cm}$ defect (Figure 4).

The defect was repaired using combined closure methods since it affected multiple subunits. The cheek and nasal sidewall were closed primarily, leaving the inferior portion to heal by second intention. The ala had its remaining portion removed and was restored with a nasolabial interpolation flap associated with a cartilage graft (Figure 5)

After three weeks, the patient was submitted to the second stage and, after two months postoperatively, the patient had an optimal result (Figure 6).

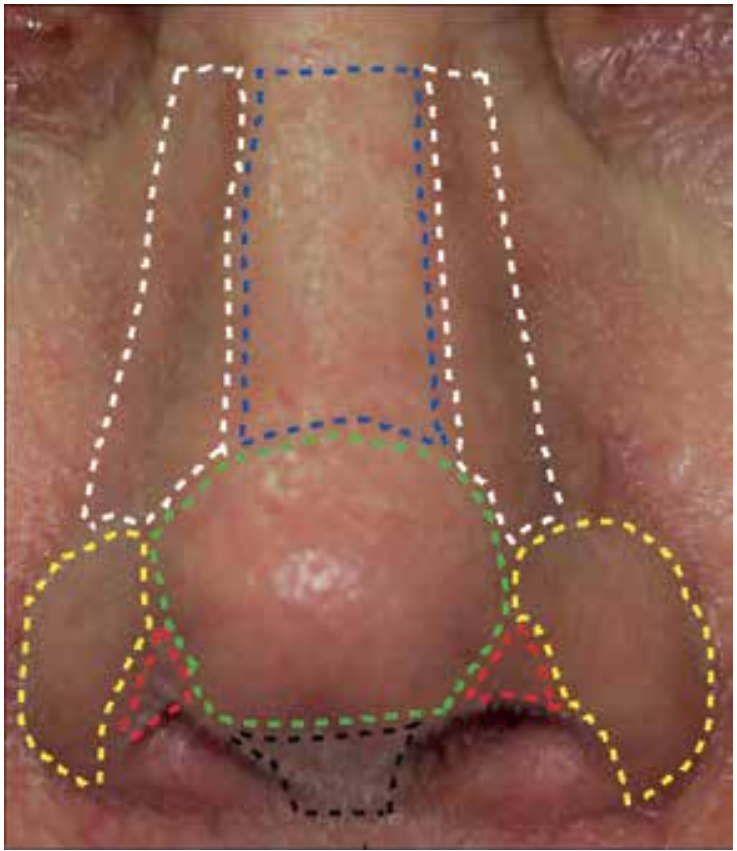

Figure 1: The nasal subunits demarcated by dotted lines: dorsum (blue), nasal sidewalls (white), tip (green), alae (yellow), soft triangles (red) and columella (black)
All procedures were performed under local anesthesia combined with infraorbital nerve block.

\section{DISCUSSION}

The nose is a complex structure that can be divided in nine subunits. The upper two thirds contain the dorsum and nasal sidewalls, whereas the lower third is composed of the columella, nasal tip, paired alae and soft triangles. ${ }^{2}$ The subunit principle was described in 1985 by Burget and Menick, and consists in replacing the entire subunit rather than simply patching the defect when a large portion of a subunit has been removed. This allows for scars to be camouflaged

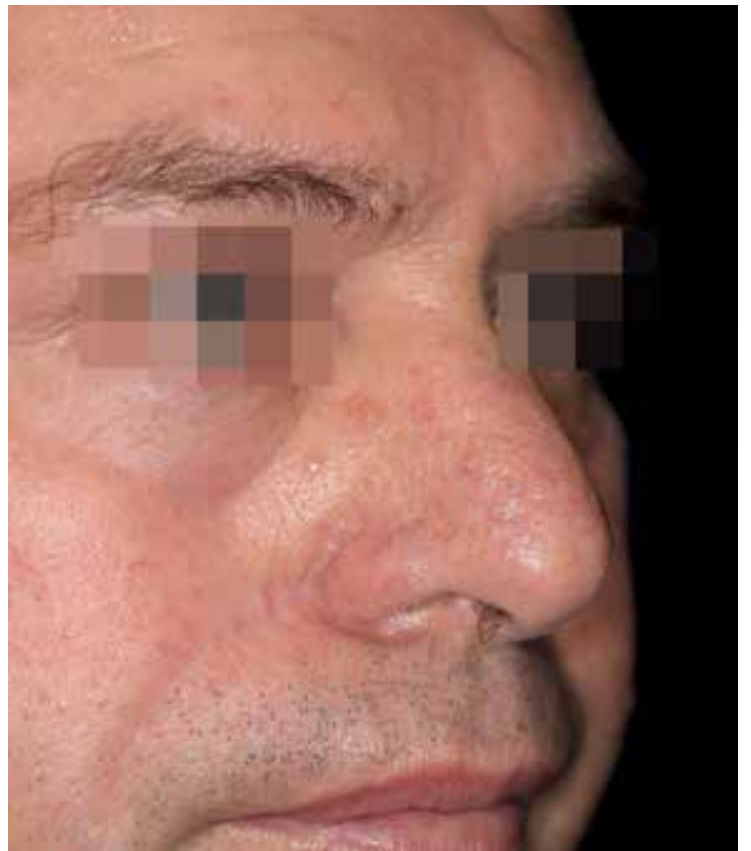

Figure 3: Nine months postoperatively. Note incision lines hidden between the limits of the nasal subunits. Also note the adequate recreation of the alar crease
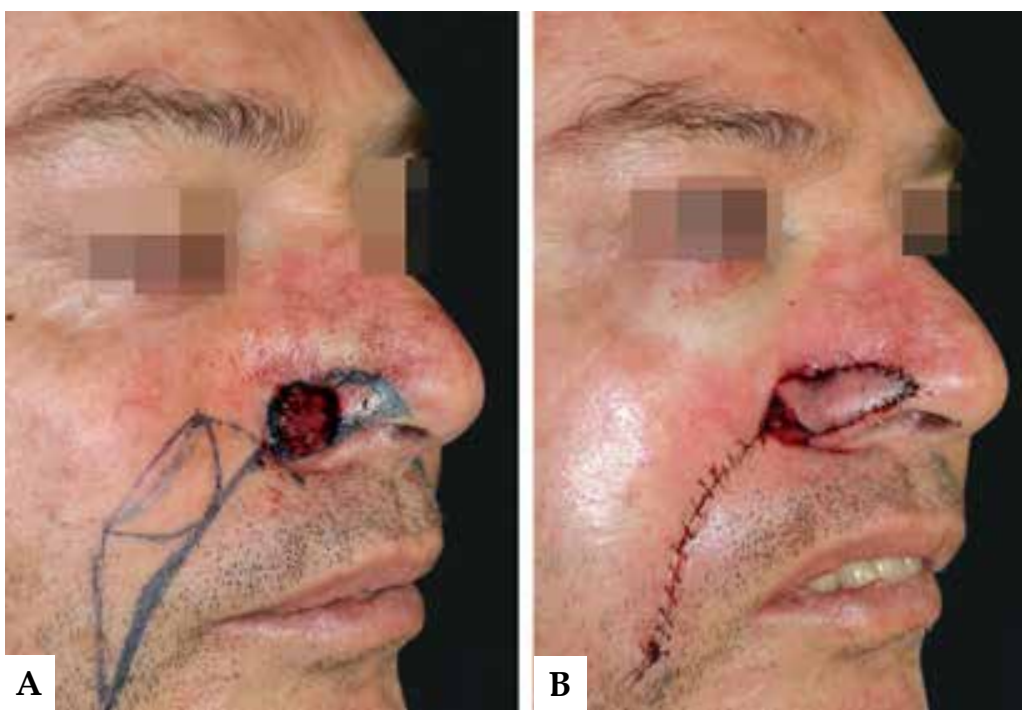

Figure 2: A - Surgical defect affecting $70 \%$ of the right ala. Nasolabial interpolation flap design. Note demarcation of the remaining subunit to be excised. B - Immediate postoperative ( $1^{\text {st }}$ stage) 

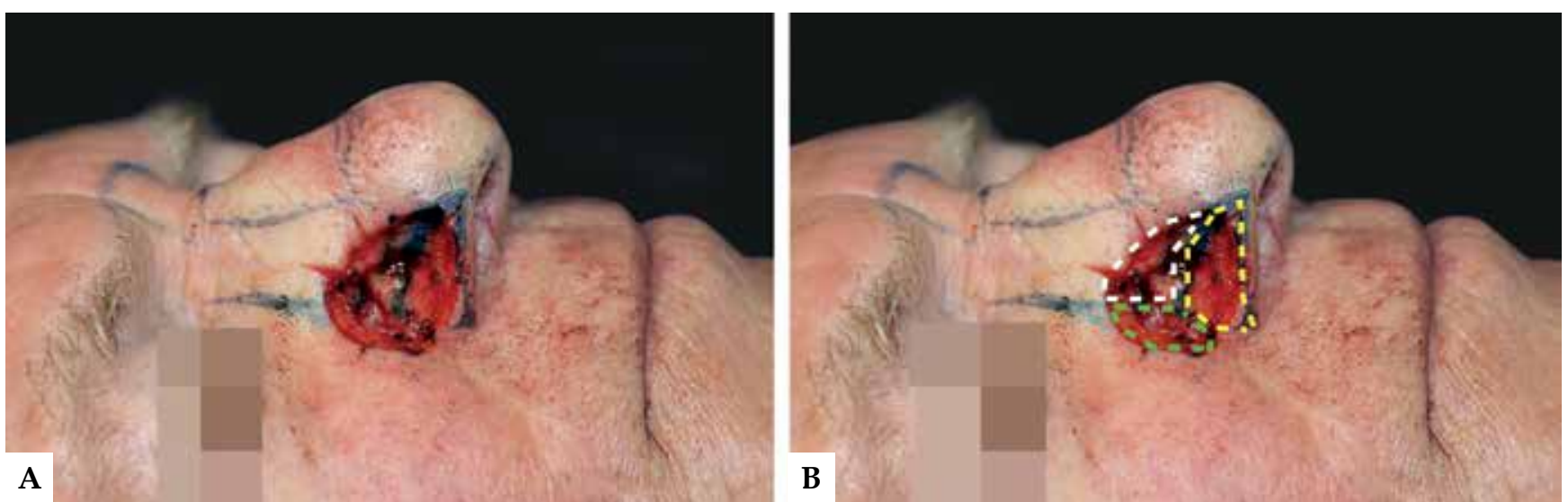

Figure 4: A - Surgical defect affecting $90 \%$ of the right ala, inferior portion of the nasal sidewall and medial cheek. The remaining portions of the ala are marked to be removed. B - Parts of the different subunits are demarcated for adequate planning: nasal sidewall (white), medial cheek (green) and nasal ala (yellow)

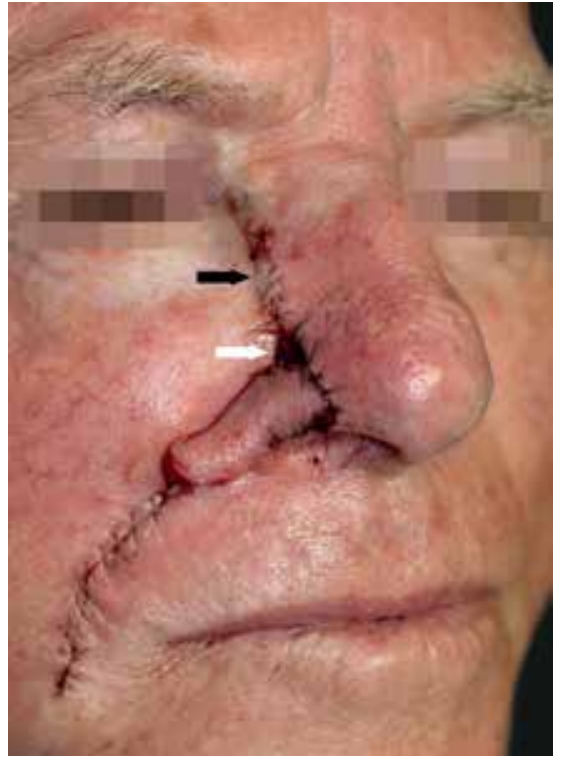

Figure 5: Immediate postoperative of nasolabial interpolation flap ( $1^{\text {st }}$ stage $)$. Nasal sidewall and cheek were closed primarily (black arrow) leaving the inferior portion to heal by second intention (white arrow)
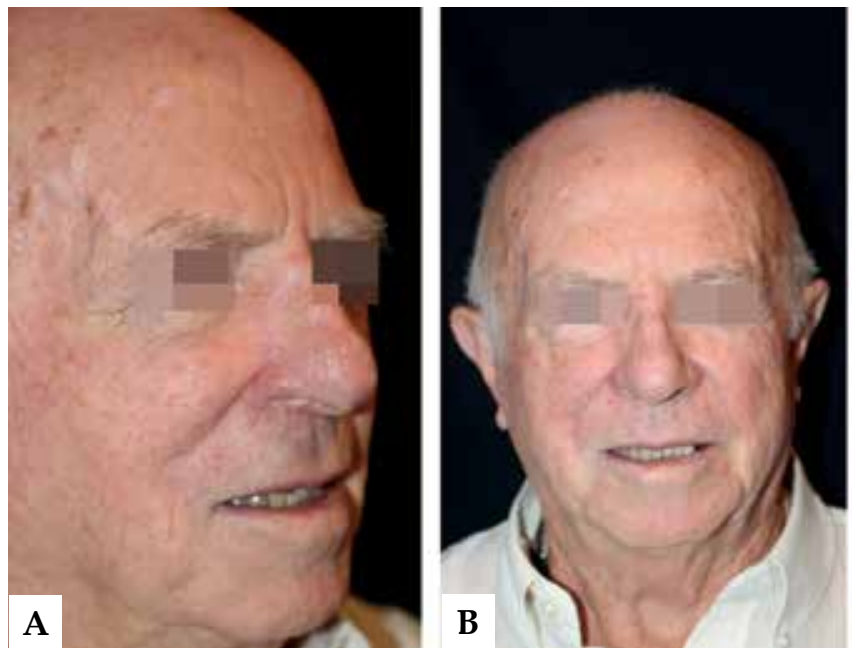

on the contours of the nasal surface, providing a superior result. Furthermore, as trapdoor contraction occurs, the entire reconstructed subunit bulges in a way that simulates the normal contour of a nasal tip, dorsum, or ala. ${ }^{1}$ For better visualization and assessment of the defect, it is important to demarcate the subunits during surgery. If sterile pens are not available, this can be performed using a "syringe pen", easily made with syringe, toothpick and methylene blue. ${ }^{3}$

Although the subunit principle is essential in nasal reconstruction, many authors have proposed reasonable modifications such as half subunit replacement, achieving very good results. ${ }^{4,5}$ In addition, several authors have demonstrated equally good results by performing reconstruction of the defect itself, instead of with a subunit approach. ${ }^{6,7}$ As there are appropriate candidates for either approach, the choice should be made based on each single case. ${ }^{2} \mathrm{Ad}-$ herence to the subunit principle is more important in the lower third of the nose, whereas defect-only reconstruction is certainly reasonable at the nasal sidewalls and dorsum. ${ }^{2}$ Other esthetic considerations, such as skin texture, color, contour, and actinic damage, are also crucial in achieving an optimal result. ${ }^{6}$ It is worth to remember that "cure comes before esthetics", and Mohs micrographic surgery has the highest cure rate for non-melanoma skin cancer. ${ }^{8}$ For this reason, if indicated, it should be performed whenever possible, be-

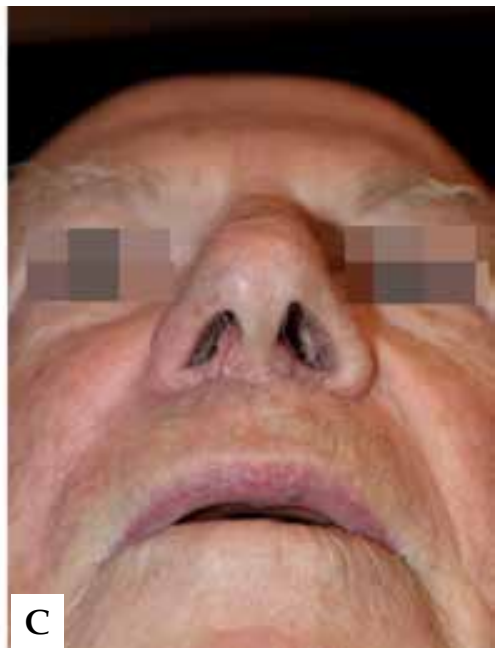

Figure 6: Two months postoperatively. A - Oblique view.

B - Frontal view. C - Inferior view. Note adequate patency of the nasal vestibule

Source: Cerci FB, 2015. ${ }^{10}$ 
fore restoring the entire subunit. While $100 \%$ of the surgical margins are evaluated during Mohs surgery, only about $1 \%$ is analyzed after regular excision. ${ }^{9}$

In the first case, other closure methods were considered including a nasolabial transposition flap. However, surgical defects located on the lateral portion of the ala should be carefully assessed since our eyes perceive a distinct separation between the nose and the cheek due to the alar crease. When blunted, the resulting asymmetry can be easily noted. Despite adequate technique, a nasolabial transposition flap would not have been able to recreate the alar crease as the nasolabial interpolation flap did. Furthermore, the nasolabial interpolation flap was able to replace the entire ala, providing a more natural appearance. The nasolabial interpolation flap, however, has a few disadvantages including two stages for its completion and pedicle care for three weeks.

\section{REFERENCES}

1. Burget GC, Menick FJ. The subunit principle in nasal reconstruction. Plast Reconstr Surg. 1985;76:239-47.

2. hornton JF, Griffin JR, Constantine FC. Nasal reconstruction: an overview and nuances. Semin Plast Surg. 2008;22:257-68.

3. Dellatorre G, Cerci FB. Syringe pen: An alternative skin-marking tool in dermatologic surgery. J Am Acad Dermatol. 2015;73:e179-80.

4. Jellinek NJ, Nguyen TH, Albertini JG. Paramedian forehead flap: advances, procedural nuances, and variations in technique. Dermatol Surg. 2014;40:S30-42.

5. Cerci FB, Nguyen TH. Paramedian forehead flap for complex nasal defects following Mohs micrographic surgery. Surg Cosmet Dermatol. 2014;6:17-24.

6. Rohrich RJ, Griffin JR, Ansari M, Beran SJ, Potter JK. Nasal reconstruction-beyond aesthetic subunits: a 15-year review of 1334 cases. Plast Reconstr Surg. 2004;114:1405-16

7. Singh DJ, Bartlett SP. Aesthetic considerations in nasal reconstruction and the role of modified nasal subunits. Plast Reconstr Surg. 2003;111:639-48.

8. Rowe DE, Carroll RJ, Day CL Jr. Long-term recurrence rates in previously untreated (primary) basal cell carcinoma: implications for patient follow-up. J Dermatol Surg Oncol. 1989;15:315-28.

9. Kimyai-Asadi A, Goldberg LH, Jih MH. Accuracy of serial transverse crosssections in detecting residual basal cell carcinoma at the surgical margins of an elliptical excision specimen. J Am Acad Dermatol. 2005:53:469-74.

10. Cerci FB. Auricular cartilage graft for nasal reconstruction after Mohs micrographic surgery. Surg Cosmet Dermatol 2015;7:109-15.
In the second case, multiple subunits were affected. When that happens, independent methods of closure should be considered. ${ }^{5}$ This is particularly true for subunits divided by concavities such as the alar crease. An attempt to cover the ala and medial cheek/nasal sidewall with one flap may result in a larger ala and/ or blunting of the alar crease. Small adjacent defects in these areas may be closed primarily, leaving the inferior portion to heal by second intention. ${ }^{10}$ This helps recreate the concavity of the crease like in the present case.

The subunit principle is an important concept for those who perform nasal reconstruction after skin cancer removal. It should be considered especially for defects located on the inferior third of the nose that affect more than $50 \%$ of a subunit.]
MAILING ADDRESS:

Felipe Bochnia Cerci

Praça Rui Barbosa, 245

Centro

80010-030 Curitiba, PR

E-mail:cercihc@hotmail.com

How to cite this article: Cerci FB. Usefulness of the subunit principle in nasal reconstruction. An Bras Dermatol. 2017;92(5 Suppl 1):159-62. 